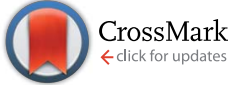

Cite this: J. Mater. Chem. B, 2015, 3 , 2522

Received 25th January 2015

Accepted 10th February 2015

DOI: $10.1039 / c 5 t b 00180 c$

www.rsc.org/MaterialsB

\section{A size dependent evaluation of the cytotoxicity and uptake of nanographene oxide $\uparrow$}

\author{
Rafael Gregorio Mendes, ${ }^{a}$ Britta Koch, ${ }^{a}$ Alicja Bachmatiuk, ${ }^{\text {abcd }}$ Xing Ma, ${ }^{\text {e }}$ \\ Samuel Sanchez, ${ }^{\text {efg }}$ Christine Damm, ${ }^{\text {a }}$ Oliver G. Schmidt, ${ }^{\text {ah }}$ Thomas Gemming, ${ }^{\text {a }}$ \\ Jürgen Eckert ${ }^{\text {aij }}$ and Mark H. Rümmeli*bd
}

\begin{abstract}
Graphene oxide (GO) has attracted great interest due to its extraordinary potential for biomedical application. Although it is clear that the naturally occurring morphology of biological structures is crucial to their precise interactions and correct functioning, the geometrical aspects of nanoparticles are often ignored in the design of nanoparticles for biological applications. A few in vitro and in vivo studies have evaluated the cytotoxicity and biodistribution of GO, however very little is known about the influence of flake size and cytotoxicity. Herein, we aim at presenting an initial cytotoxicity evaluation of different nano-sized GO flakes for two different cell lines (HeLa (Kyoto) and macrophage (J7742)) when they are exposed to samples containing different sized nanographene oxide (NGO) flakes (mean diameter of 89 and $277 \mathrm{~nm}$ ). The obtained data suggests that the larger NGO flakes reduce cell viability as compared to smaller flakes. In addition, the viability reduction correlates with the time and the concentration of the NGO nanoparticles to which the cells are exposed. Uptake studies were also conducted and the data suggests that both cell lines internalize the GO nanoparticles during the incubation periods studied.
\end{abstract}

\section{Introduction}

Nanotechnology has provided the means to synthesize different types of nanoparticles from a wide variety of materials, with

${ }^{a}$ Institute for Solid State and Materials Research Dresden, Helmholtzstr. 20, 01069, Dresden, Germany. E-mail: m.ruemmeli@ifw-dresden.de

${ }^{b} I B S$ Center for Integrated Nanostructure Physics, Institute for Basic Science (IBS), Daejon 305-701, Republic of Korea

${ }^{c}$ Centre of Polymer and Carbon Materials, Polish Academy of Sciences, M. CurieSklodowskiej 34, Zabrze 41-819, Poland

${ }^{d}$ Department of Energy Science, Department of Physics, Sungkyunkwan University, Suwon 440-746, Republic of Korea

${ }^{e}$ Max Planck Institute for Intelligent Systems, Heisenbergstr. 3, Dresden, 70569, Stuttgart, Germany

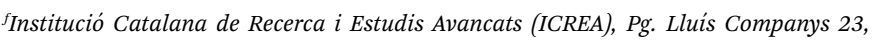
08010, Barcelona, Spain

${ }^{g}$ Institut de Bioenginyeria de Catalunya (IBEC), Baldiri Reixac 10-12, 08028, Barcelona, Spain

${ }^{h}$ Material Systems for Nanoelectronics, Chemnitz University of Technology, Reichenhainer Str. 70, 09107 Chemnitz, Germany

institute of Materials Science TU Dresden, 01062 Dresden, Germany

${ }^{j}$ Center for Advancing Electronics Dresden (cfaed), TU Dresden, 01062 Dresden, Germany

$\dagger$ Electronic supplementary information (ESI) available: Fig. S1: aqueous dispersion of NGO; Fig. S2: histograms showing the measurements of diameter and height of NGO; Fig. S3: Raman and Infrared spectra of NGO; Fig. S4: EDX measurement of NGO done in the TEM; Fig. S5: XPS spectra of NGO; Fig. S6: optical images for trypan blue assay of HeLa cells and Fig. S7: optical images for trypan blue assay of macrophages; Fig. S8: uptake images of iron oxide NPs after 48 hours incubation. See DOI: 10.1039/c5tb00180c each pursuing a diverse range of features (e.g. size, shape and surface reactivity). Only after thorough characterization of their morphological features is it possible to individually study what the actual role of the nanoparticles is when in contact with biological systems. This is also crucial to draw accurate correlations between the nanoparticle design and a specific biological response and ultimately use these data to build a database containing information on how specific features of nanoparticles are linked to specific biological responses. ${ }^{1,2}$

The inspiration to create such a database is linked to natural design. Molecules, viruses, bacteria and other biological structures have evolved into precise sizes, shapes and chemistries to mediate specific interactions and functions. ${ }^{2}$ Viruses, for example, pursue capsids with different symmetries such as icosahedral, bullet-shaped, rod-shaped or can have asymmetric morphologies. These geometries are thought to play an important role in their ability to infect specific cell types and may alter their residence time inside the cell. ${ }^{2-4}$

To date, the physicochemical properties of naturally occurring nano-sized complexes and structures are far from being understood. However, it is clear that these biological structures follow specific design rules. ${ }^{5}$ Hence, in order to engineer nanostructures capable of specific functions, such as to circulate in the body, infect and control cells or to detect and repair unhealthy cells, it is crucial to understand how synthetic nanostructured materials interact with biological systems.

The different shapes, sizes and properties of nanostructured materials are not only thought to have many different 
biomedical applications, ${ }^{6-9}$ but also present different cytotoxic potential. ${ }^{10,11}$ A recent study showed that the binding and activation of membrane receptors strongly depend on the size of silver and gold nanoparticles. ${ }^{12}$ This provides evidence that morphological aspects of nanoparticles can mediate molecular processes that are crucial for regulating the correct function of the cell. Indeed, different diameters of carbon nanotubes show different interactions with proteins, which can disturb vital cellular functions. ${ }^{\mathbf{1 3}}$

The two dimensional $\mathrm{sp}^{2}$ hybridized carbon structure graphene presents extraordinary properties in the field of material science and physics. It initially attracted interest because of its mechanical, optical and electronic properties, ${ }^{14-17}$ but graphene is also thought to have biological application in fields such as biosensing. ${ }^{18}$ However, since graphene is highly hydrophobic, its use for biological purposes is more promising in its oxidized form, namely graphene oxide (GO). The GO provides a better platform for biological purposes because it is hydrophilic due to the presence of different functional groups on its surface and edges. Therefore, GO can be easily dispersed and stabilized in a physiological environment. These functional groups together with the high loading capacity render GO a unique platform for further functionalization with drugs, polymers and other biomolecules. ${ }^{19-21}$

In fact, it has been already well reported in the literature that the functionalization of graphene and graphene oxide flakes with polymers ${ }^{22,23}$ and other biomolecules ${ }^{24}$ increase their dispersion and stability in a wide variety of physiological media as well as render additional properties to the material. Dai et $a{ }^{25}$ used polyethylene glycol (PEG) for the first time to functionalize GO, which also served as an anchor for further anticancer drugs such as doxorubicin. ${ }^{25}$ The conjugation of drug and GO leaded to a significant enhancement of chemotherapy efficacy. ${ }^{26}$ However, despite the functionalization which yields novel and useful properties it has also been reported that slight changes in the molecules used to functionalize graphene oxide can cause different biological responses, e.g. GO functionalized with the linear PEG is more internalized than a branched version of PEG. $^{22}$ Moreover, the branched PEG induces more cellular function changes than its linear counterpart. Hence it is firstly of interest to study GO without the influence of extra functionalization and correlate any response with flake size.

Despite the promising potential of GO to be further tailored with specific biomolecules, ${ }^{27}$ very little is known about the influence and correlation of the NGO size and the different biological responses it might trigger on cells, e.g. toxicity, uptake. De Marzi et al. ${ }^{28}$ showed recently, evidence that graphene oxide flakes have a high genotoxic response on different cell lines. Flakes with sub $100 \mathrm{~nm}$ present the highest genotoxic.

Furthermore, a few in vitro studies have already been conducted to evaluate the biocompatibility of graphene oxide ${ }^{29}$ in different cell lines. Recently in vivo studies have been conducted to evaluate the biodistribution and of GO in mice, ${ }^{30,31}$ but none of these studies addressed any possible correlation between the size of the GO flakes with the biological response studied.
Carbon black (CB) nanoparticles are more discussed in the literature. A recent work using CB nanoparticles with different sizes (50 $\mathrm{nm}$ and $500 \mathrm{~nm}$ ) induce inflammatory responses in THP-1 cells. The smaller nanoparticles altered the phagocytic capacity of cells while no significant alteration could be seen for the big nanoparticles. ${ }^{32}$ Another study with CB nanoparticles showed that large sizes are more internalized by cells. ${ }^{33}$

Herein, we synthesized two different samples, each containing nano-sized graphene flakes with distinct mean size distributions and investigated the viability of two different cell lines when exposed to these two nanographene oxide (NGO) sizes. The study was conducted for different incubation periods and our results suggest that both the size of the nanographene oxide flakes and the incubation period play a role in the toxic effect triggered by these nanoparticles. The uptake of the nanographene oxide was also investigated and showed that cells can internalize the nanoparticles during the incubation period studied.

\section{Materials and methods}

\subsection{Synthesis}

The nanographene oxide (NGO) was produced from commercially available natural graphite and was based on a modified Hummers method..$^{\mathbf{2 0 , 3 4 , 3 5}}$ Briefly, $1 \mathrm{~g}$ of the commercially available graphite was ground with $50 \mathrm{~g}$ of sodium chloride $(\mathrm{NaCl})$ for 15 minutes in order to exfoliate the graphite as well as reduce its size. In order to remove the $\mathrm{NaCl}$ mixed with graphite, the contained $\mathrm{NaCl}$ was dissolved in distilled water at a temperature of $c a .55{ }^{\circ} \mathrm{C}$ and subsequently filtrated using $450 \mathrm{~nm}$ porous TEFLON filter paper by suction filtration. The filtrated graphite was mixed in $23 \mathrm{~mL} 95 \%$ sulfuric acid $\left(\mathrm{H}_{2} \mathrm{SO}_{4}\right)$ and left it stirring vigorously overnight. Afterwards, the temperature of the mixture was kept constant at $10^{\circ} \mathrm{C}$ in an ice bath while $3 \mathrm{~g}$ potassium permanganate $\left(\mathrm{KMnO}_{4}\right)$ was added gradually over $3 \mathrm{~h}$ with constant stirring. Afterwards, the mixture was sonicated for $3 \mathrm{~h}$ and continuously stirred for 30 minutes at $35{ }^{\circ} \mathrm{C}$ and another 45 minutes at $50{ }^{\circ} \mathrm{C}$. Finally, $46 \mathrm{~mL}$ distilled water was added to the mixture and kept stirring at $100{ }^{\circ} \mathrm{C}$ for $45 \mathrm{~min}$. The mixture was cooled down to room temperature and stirred with the addition of $140 \mathrm{~mL}$ distilled water and $10 \mathrm{~mL}$ of $30 \%$ hydrogen peroxide $\left(\mathrm{H}_{2} \mathrm{O}_{2}\right)$. The final solution filtrated and washed 10 times with $5 \% \mathrm{HCl}$ and distilled water to remove any reaction byproducts and the oxidized graphene was collected. In order to further reduce both the width (lateral cracking) and thickness (exfoliation) of the oxidized graphene flakes, distilled water was added and the solution was then subjected to ultrasonication using a horntipped ultrasonic probe. In this work two different sizes of NGO were produced (depending on the sonication period). Tipsonication for 30 minutes produced samples with an average NGO flake size of $277 \mathrm{~nm}$ and $\sim 4$ layers (ca. $1.4 \mathrm{~nm}$ ), while was tip-sonication for 4 hours reduced the average flake size to $89 \mathrm{~nm}$ and $\sim 6$ layers (ca. $2.1 \mathrm{~nm})$. 


\subsection{Sample characterization}

Diameter distribution. A droplet of the aqueous solution containing the NGO was dried on a clean silicon ( $\mathrm{Si}$ ) wafer. The Si wafer containing the NGO was then observed in the scanning electron microscope (FEI NOVA NanoSEM200). The images containing the flakes were then analyzed with statistical methods. For each sample, more than one hundred particles were measured at least four times each. The average of the measurements was taken as the mean diameter of the flake.

Number of graphene layers. In order to obtain the average number of graphene layers, the samples on the Si wafers carrying the NGO were measured using an atomic force microscope (Digital Instruments Veeco, NanoScope IIIa) operating in the tapping mode. Thereby it was possible to obtain information about the topography of the sample, namely the height of individual NGO flakes. Since it is known that the space between individual layers is approximately $0.35 \AA$, it is possible to estimate the number of layers in each measured flake and hence also plot a distribution. In this case each individual flake was measured at least four times for over one hundred particles. These measurements were then averaged. For more details on sample preparation as well as on size and the number of graphene layers see Fig. S1 in the ESI. $\dagger$ To validate the mean size of the flakes in an aqueous solution, dynamic light scattering (Malvern Instruments Ltd.) was performed at room temperature.

$\mathbf{s p}^{2}$ hybridization and functional groups. The presence of $\mathrm{sp}^{2}$ carbon was confirmed using Raman spectroscopy (Thermo Scientific DXR, laser $=532 \mathrm{~nm}$ ), while the presence of functional groups was confirmed by the use of Fourier transform infrared spectroscopy (Bruker IFS 113 spectrometer) and X-ray photoemission spectroscopy (XPS) equipped with a hemispherical electron analyzer SPECS PHOIBOS 100.

\subsection{Biological characterization}

Cell culture. HeLa cells (Kyoto line) and macrophages J7442 cell. The HeLa cells were cultured in DMEM+GlutaMAXTM (gibco) supplemented with 1\% NEAA (gibco); macrophage J7742 cells were grown in DMEM F12HAM supplemented with $2 \mathrm{mM}$ L-glutamine (Sigma-Aldrich). Additionally, all the media contained 10\% FBS (Sigma-Aldrich), $100 \mathrm{U} \mathrm{mL}^{-1}$ penicillin and 100 $\mu \mathrm{g} \mathrm{mL} \mathrm{m}^{-1}$ streptomycin (gibco). To create a cell suspension the cells were washed once with PBS (gibco) and detached using a cell scraper for the macrophages (Greiner Bio-One) or for the other cell line, $0.25 \%$ Trypsin-EDTA solution (Sigma).

MTT viability assay. For cell viability studies, a commercial TOX-1 in vitro toxicology assay kit (Sigma-Aldrich) was used. The assay measures the viability of living cells via the cleavage of MTT (3-[4,5-dimethylthiazol-2-yl]-2,5-diphenyltetrazolium bromide) to purple formazan crystals by the cells' mitochondrial dehydrogenases. Two different cell lines were grown in standard 96 well plates as testing systems and $5.0 \times 10^{3}$ cells were seeded per well.

The NPs were suspended in DI water at a stock concentration of $0.5 \mathrm{mg} \mathrm{mL}^{-1}$. To assure a uniform dispersion of the particles the suspensions were vortexed (stirred) on a Vortex Genie $2 \mathrm{~T}$ for 2 min prior to usage. After incubating the cells at $37{ }^{\circ} \mathrm{C}$ and $5 \%$ $\mathrm{CO}_{2}$ in the presence of increasing concentrations of NPs (controls with only DI water, $1 \mu \mathrm{g} \mathrm{mL} L^{-1}, 10 \mu \mathrm{g} \mathrm{mL}^{-1}$ and $100 \mu \mathrm{g} \mathrm{mL}{ }^{-1}$ ) for either 12 or $48 \mathrm{~h}, 10 \mu \mathrm{L}$ of the MTT solution ( $5 \mathrm{mg} \mathrm{mL}^{-1}$ in PBS) was added to the $0.1 \mathrm{~mL}$ culture medium per well and the 96 well plates were returned to the incubator for another $4 \mathrm{~h}$. After carefully removing $80 \mu \mathrm{L}$ of the supernatant the produced formazan crystals were then dissolved in $0.1 \mathrm{~mL}$ of the pre-warmed MTT solubilization solution (10\% Triton X-100, $0.1 \mathrm{M} \mathrm{HCl}$ in anhydrous isopropanol) by gently shaking the plates in an ELISA microplate reader ( $15 \mathrm{~min}, 14 \mathrm{~Hz}, 37{ }^{\circ} \mathrm{C}$, SAFAS MP96). The absorbance of the formazan dye was measured in the SAFASMP96 reader at $550 \mathrm{~nm} / 650 \mathrm{~nm}$ within an hour and after having subtracted the background from the sample, the percentage cell viability was calculated. Each of the conducted experiments was repeated three times. Both nanoparticle- and cell-free controls were performed. The statistical analysis was conducted using an ordinary one-way ANOVA followed by Bonferroni multiple comparisons post-test with GraphPad InStat 3 (Version 3.10, 32 bit, GraphPad Software, Inc., La Jolla, USA). A value of $p<0.05$ was considered as statistically significant.

Dye exclusion test (trypan blue). The cell lines were grown in standard 6 well plates and incubated for 12 or $48 \mathrm{~h}$ with the respective nanoparticle suspension at a concentration of $10 \mu \mathrm{g} \mathrm{mL}{ }^{-1}$. Afterwards the cells were detached and resuspended in $1 \mathrm{~mL}$ cell culture Medium per well. $20 \mu \mathrm{L}$ of each cell suspension were carefully mixed with $0.4 \%$ trypan blue solution (Sigma) and added to the chambers of a standard hemocytometer (improved Neubauer) to count the number of viable and dead cells. The results are presented as means of two measurements each and their deviation.

TEM investigations. The cells were grown as a monolayer in a humidified incubator in the conditions described above. The cells were seeded in a $6 \mathrm{~cm}$ Petri dish for $4 \mathrm{~h}$ prior to the incubation with the NGO. After this adhesion period the bottom the cells were treated with the NPs in a concentration of $10 \mu \mathrm{g} \mathrm{mL} \mathrm{m}^{-1}$ for a period of $48 \mathrm{~h}$. The cells were collected from the culture dish and centrifuged at $1000 \mathrm{rpm}$ for $5 \mathrm{~min}$, and the supernatant was removed. The cell pellets were fixed in a $2.5 \%$ gluteraldehyde solution for $4 \mathrm{~h}$. They were then rinsed with PBS and embedded in a $4 \%$ agarose gel. A second fixation step was conducted in $2 \%$ osmium tetroxide solution for $24 \mathrm{~h}$ and then rinsed with distilled water. The samples were stained with $1 \%$ uranyl acetate for $1.5 \mathrm{~h}$, dehydrated in a graded series of acetone $(25,50,75,96$ and 100\%), and embedded in epoxy resin. First, the resin was polymerized for $3 \mathrm{~h}$ at $50{ }^{\circ} \mathrm{C}$ and then the temperature increase to $60{ }^{\circ} \mathrm{C}$ for $48 \mathrm{~h}$. Ultrathin sections $(50-70 \mathrm{~nm})$ were obtained with an ultramicrotome (Leica EM UC7), transferred to a TEM grid and imaged using a transmission electron microscope (TEM) FEI TECNAI T20 operating at $200 \mathrm{kV}$.

\section{Results}

As illustrated in Fig. 1, two different sizes of NGO flakes were synthesized and characterized prior to the biological evaluation. The size-dependent cytotoxicity was investigated using the MTT 


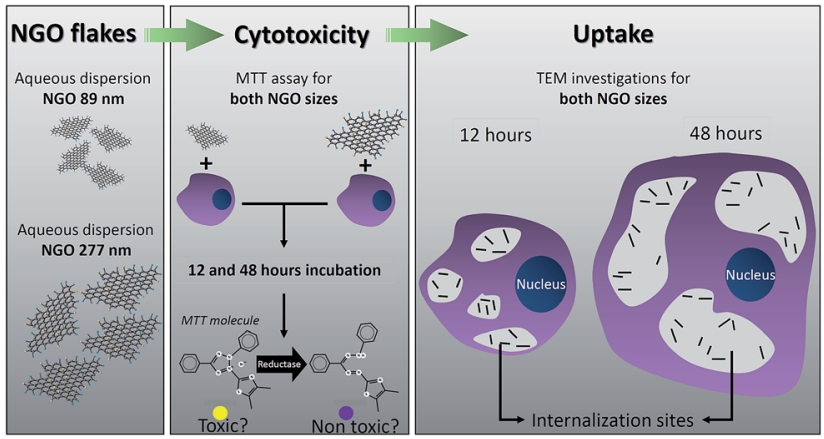

Fig. 1 Schematic illustrating the procedure employed to evaluate the size-dependent cytotoxicity and cellular uptake of NGO.

assay for 12 and 48 hours incubation periods. Additionally, the cell death was assessed through the trypan blue assay. The uptake was studied using a transmission electron microscope (TEM) of $70 \mathrm{~nm}$ thick slices of cells embedded in epoxy resin.

\subsection{Nanoparticle synthesis and characterization}

The morphology of the two as-produced nanographene oxide samples was studied using various techniques. In Fig. 2 panel (a) to (c) provide typical SEM, AFM and TEM images of the nanographene sample containing flakes with a mean diameter of $277 \mathrm{~nm}$ and $c a .4$ graphene layers. In Fig. 2 panel (d) to (f) similar SEM, an AFM and a TEM images of the nanographene sample containing flakes with a mean diameter of $89 \mathrm{~nm}$ and ca. 6 graphene layers are provided. Pictures of the solution containing the concentrated nanographene samples in aqueous solution is shown in Fig. S2 in the ESI. $\dagger$ The flake sizes obtained by SEM imaging is in agreement with dynamic light scattering (DLS) data of NGO in aqueous solution. In this case a concentrated solution of NGO was diluted to concentrations of 1,10 and $100 \mu \mathrm{g} \mathrm{mL} \mathrm{m}^{-1}$. The obtained hydrodynamic sizes are higher than those under dried conditions (as expected due to

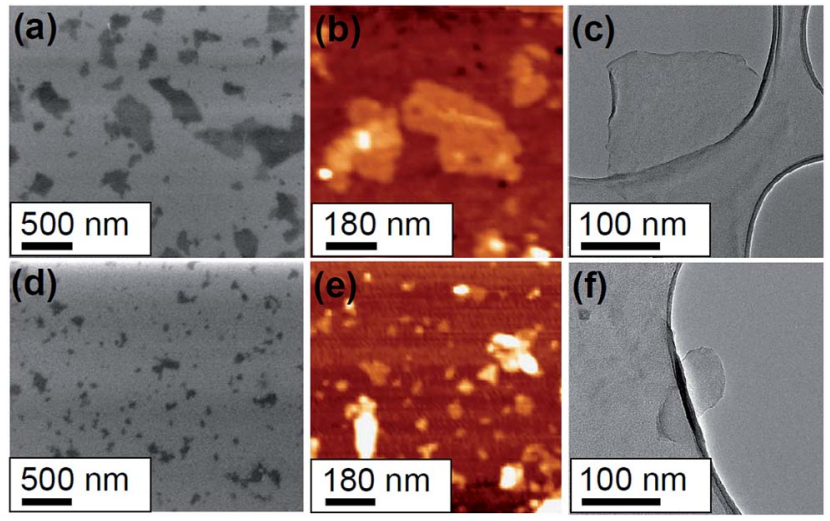

Fig. 2 Panel (a) shows the SEM image of NGO with $277 \mathrm{~nm}$ in diameter and panel (b) shows an AFM image where the measured height of NGO was 4 layers. Panel (c) shows a TEM image of the panels (d) to (f) show the same set of measurements for a second sample containing NGO nanoparticles with an average diameter of $89 \mathrm{~nm}$ and 6 layers.
Table 1 DLS size in aqueous solution

\begin{tabular}{lcc}
\hline Sample & Concentration $\left[\mu \mathrm{g} \mathrm{mL}^{-1}\right]$ & DLS size \pm error $[\mathrm{nm}]$ \\
\hline $1(277 \mathrm{~nm})$ & 1 & $489.9 \pm 34.6$ \\
& 10 & $394.8 \pm 3.8$ \\
$2(89 \mathrm{~nm})$ & 100 & $399.26 \pm 10.9$ \\
& 1 & $112.9 \pm 1.5$ \\
& 10 & $107.5 \pm 3.6$ \\
& 100 & $98.3 \pm 2.4$
\end{tabular}

hydration). The DLS results for both samples in aqueous solutions are shown in Table 1.

The presence of $\mathrm{sp}^{2}$ carbon hybridization was confirmed by Raman spectroscopy as shown in Fig. S3 in the ESI. $\uparrow$ The Raman data shows the presence of the $\mathrm{D}, \mathrm{G}$ and $2 \mathrm{D}$ modes at $c a$. $1350 \mathrm{~cm}^{-1}, 1600 \mathrm{~cm}^{-1}$ and $2700 \mathrm{~cm}^{-1}$ respectively, which are characteristic signatures for graphene. The presence of a large D mode indicates that the graphene flakes are rather defective. This is expected since the initial graphite was oxidized and different functional groups were inserted both on the basal planes and edges of the nanographene flakes. The presence of functional groups were confirmed using Fourier transform infrared spectroscopy (FTIR). The IR spectra are presented in Fig. $\mathrm{S} 3 \dagger$ and present a variety of functional species. The most characteristic groups show peaks at 750, 1700 and $2225 \mathrm{~cm}^{-1}$, which correspond to $\mathrm{C}-\mathrm{O}, \mathrm{C}-\mathrm{H}$ and $\mathrm{C}=\mathrm{O}$ groups, respectively.

In order to check the presence of contaminants or undesired materials, EDX measurements of individual flakes were performed when observing individual nanographene oxide flakes in the TEM (see Fig. S4 in the ESI†). The EDX data only shows the presence of carbon (C) and oxygen (O). The presence of a copper $(\mathrm{Cu})$ signal is attributed to the TEM Copper grids. XPS investigations were also conducted and are shown in Fig. S5 in the ESI. $\dagger$ It provides typical XPS spectra of the nanographene oxide samples for the $\mathrm{C}$ 1s (Fig. S5(a) $\dagger$ ) and for the $\mathrm{O} 1 \mathrm{~s}$ (Fig. S5(b)†). For the $\mathrm{C}$ 1s edge, deconvolution of the peak revealed various components corresponding to different functional groups at 284.4, 285.1, 286.8 and $288.6 \mathrm{eV}$, which correspond respectively to $\mathrm{sp}^{2}$ carbon, hydroxylic, epoxy, carbonyl and carboxylic functional groups. The $O$ 1s spectra were deconvoluted and showed peaks centered at 531.3, 533.3 and $535.1 \mathrm{eV}$, which correspond to carboxylic, epoxy and hydroxylic functional groups.

The XPS data shown in Fig. S5† panels (a), (b) and (c) reveal the presence of trace amounts sulfur (S), sodium (Na) and chlorine $(\mathrm{Cl})$, respectively. The presence of these elements is assigned to the initial GO synthesis process, which used acids containing these elements. As mentioned before, the samples were rinsed abundantly with distilled water in order to remove unwanted byproducts from the oxidation reaction. However even with intense rinsing some of these byproducts might penetrate in between the graphene layers, remain trapped inside or be physicochemically absorbed and are difficult to fully avoid. In this case the contaminants offer no risk since they are not free in the solution. 


\subsection{Toxicity evaluation}

The size dependent cytotoxicity of the nanographene oxide flakes was investigated. Two different cell lines (HeLa Kyoto and macrophages (J7442)) were incubated with the NGO dispersed in water at three distinct concentrations $\left(1,10\right.$ and $\left.100 \mu \mathrm{g} \mathrm{mL} \mathrm{m}^{-1}\right)$ for a period of 12 and $48 \mathrm{~h}$. In order to quantify the toxicity of the different NGO, two different approaches were used. Initially, the mitochondrial activity of the cells was assessed with a colorimetric assay that is based on the reduction of yellow MTT (3-[4,5-dimethylthiazol-2-yl]-2,5-diphenyltetrazolium bromide) to purple formazan crystals by mitochondrial dehydrogenase enzymes. The cleavage of MTT occurs only in living cells so that the amount of the produced formazan crystals is proportional to the number of living cells. In addition, to exclude the possible influence of the NPs on the test results, cell-free tests were simultaneously conducted. The MTT assay does not provide any information as to whether the NPs themselves cause cell death or whether they simply reduce the cellular metabolic activity. Hence we implemented a second approach, the dye exclusion method with trypan blue. It is based on the exclusion of the blue dye from healthy cells. In dead cells the membrane is no longer functional (ruptured) and the dye is able to stain the cell interior. Using a visible light microscope, the dead cells are observed to be dark blue instead of bright.

MTT assay. The data from the MTT assay after 12 and 48 hours exposure to NGO are provided in Fig. 3(a) and (c) for the Hela cells and in Fig. 3(b) and (d) for the macrophages, respectively. The sample containing NGO nanoparticles with 89 $\mathrm{nm}$ diameter incubated for 12 hours showed no strong difference in the mitochondrial activity for all concentrations used, whereas for the sample with $277 \mathrm{~nm}$, the mitochondrial activity for the 10 and $100 \mu \mathrm{g} \mathrm{mL}{ }^{-1}$ tests decreases. When the same set of data is compared with the incubation period of 48 hours for
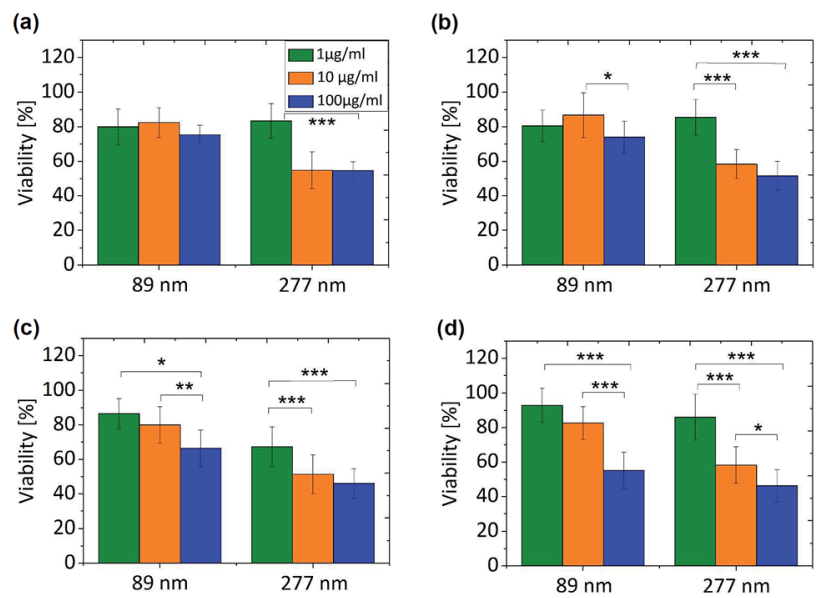

Fig. 3 Panels (a) and (c) show the MTT test using the HeLa cells incubated for $12 \mathrm{~h}$ and $48 \mathrm{~h}$ with both NGO samples, respectively. Panels (b) and (d) show the MTT test using macrophages incubated for $12 \mathrm{~h}$ and $48 \mathrm{~h}$ with both NGO samples. Note: the data was normalized to a cell density of $14.7 \times 10^{3}$ cells per $\mathrm{cm}^{2} ; *: p<0.05 ; * *: p<0.01$; ***: $p<0.001$; bars without mark mean a non-significant $p>0.05$ (statistics were obtained in one-way ANOVA followed by a Bonferroni post test). both the $89 \mathrm{~nm}$ and $277 \mathrm{~nm}$ samples the mitochondrial activity decreases with increasing concentration. This suggests that for shorter incubation periods the bigger flakes are inducing a higher toxicity and when the incubation period is increased the toxicity is similar for both sizes. The data suggest a size dependence on the toxicity for the shorter incubation periods in both cell lines in a comparable manner, whereas for longer incubation periods the concentration of the material seems to play a more important role than the size of the nanoparticles used. It is also important to mention that the controls were also measured and in all cases the viability remained at values close to $100 \%$, suggesting that only the presence of the GO nanoparticles influences the reduction of cytotoxicity values.

Dye exclusion assay (trypan blue assay). The dye exclusion test (trypan blue) was also conducted for both samples of nanographene oxide (89 $\mathrm{nm}$ and $277 \mathrm{~nm}$ ). Representative images are shown in the ESI in Fig. S6 and S7. $\uparrow$ The histograms show the viability obtained for both cell lines incubated with the nanoparticles at a concentration of $10 \mu \mathrm{g} \mathrm{mL} \mathrm{m}^{-1}$ are shown in Fig. 4. Both the HeLa cells and the macrophages do not present a decrease in viability. This indicates that the cells incubated with the nanographene oxide nanoparticles tend to undergo a reduction in mitochondrial activity (indicated by MTT assay) rather than die as indicated by the trypan blue assay in Fig. 4(a)-(d).

\subsection{Uptake evaluation}

The uptake of the nanographene oxide nanoparticles was investigated using TEM. The TEM studies were conducted for the two cell lines incubated with NPs at a concentration of $10 \mu \mathrm{g} \mathrm{mL}{ }^{-1}$. Fig. 5(a) and (b) show a HeLa cell incubated with the $89 \mathrm{~nm}$ flakes for 12 and 48 hours and Fig. 5(c) and (d) with the $277 \mathrm{~nm}$ for 12 and $48 \mathrm{~h}$, respectively. The HeLa cells internalized both samples with no obvious difference in the amount
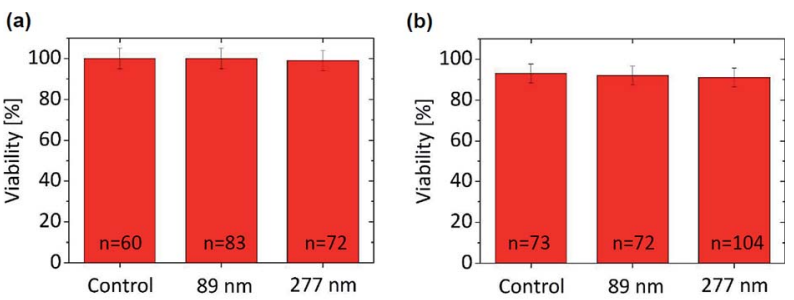

(c)

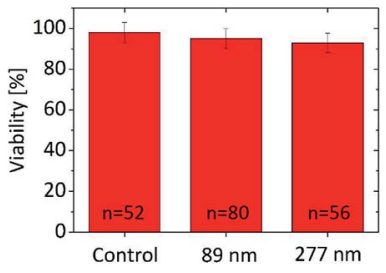

(d)

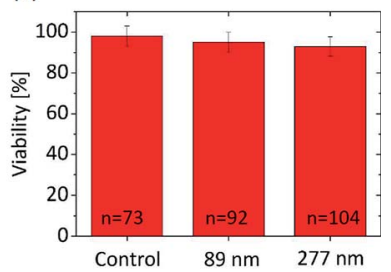

Fig. 4 Panels (a) and (c) show the trypan blue test for the HeLa cells incubated for $12 \mathrm{~h}$ and $48 \mathrm{~h}$ with both NGO samples, respectively. Panels (b) and (d) show the trypan blue test for the macrophages incubated for $12 \mathrm{~h}$ and $48 \mathrm{~h}$ with both NGO samples. The variable $n$ indicates the number of cell counts. 

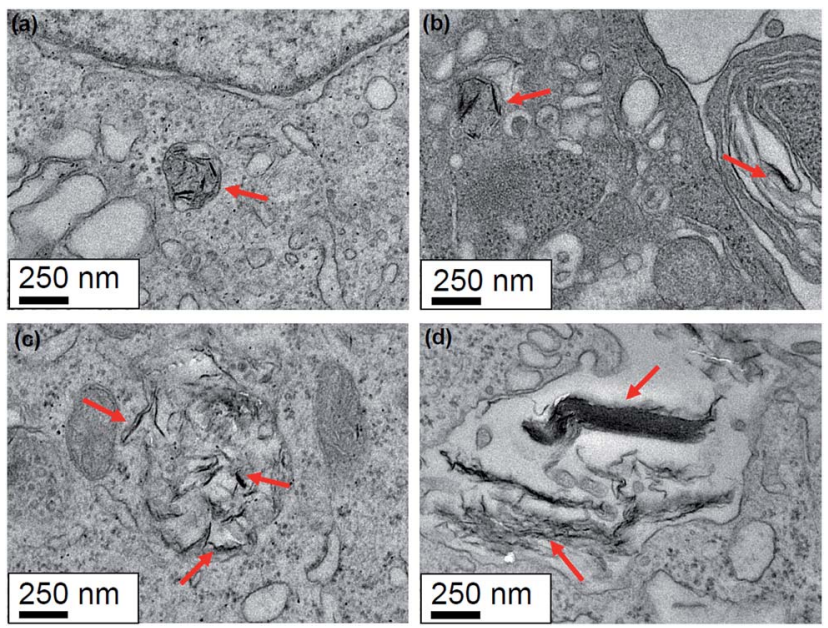

Fig. 5 In (a) and (b) it is possible to see the TEM images of the HeLa cells incubated with the $89 \mathrm{~nm}$ for 12 and $48 \mathrm{~h}$, respectively. In (c) and (d) it is possible to see the HeLa cells incubated with the $277 \mathrm{~nm}$ for 12 and $48 \mathrm{~h}$, respectively. In the TEM images it is possible to see that both sizes are internalized by HeLa cell as depicted by the arrows.

of uptaken material being determined (using TEM). The data clearly shows that the nanoparticles are compartmentalized inside vesicles and are mostly present in a many-particle fashion. This could be the result of the agglomeration of flakes in the culture medium, which is especially the case after long incubation periods as depicted in Fig. 5(d). However, inside the vesicles it is possible to see that the size of both samples match the measured values determined from the SEM images for individual particle sizes.

The results presented do not provide information on what endocytic pathway is used by the cell to internalize the NGO flakes and is the scope of the current work. For this purpose, the inhibition of specific pathways of endocytosis is necessary as recently published by Linares et al. ${ }^{36}$ The influence of flake size on the endocytic pathway was studied by Mu et al. ${ }^{37}$ and the data suggest that small flakes are internalized by clathrin-mediated endocytosis (CME), whereas big flakes are mainly internalized by phagocytosis and CME.

The same set of uptake studies were also performed for the macrophages. The observed results were similar as the HeLa cells. Fig. 6 panels (a) and (b) show a macrophage cell incubated with the $89 \mathrm{~nm}$ flakes for 12 and 48 hours, respectively, whereas in Fig. 6(c) and (d) a macrophage cell incubated with the $277 \mathrm{~nm}$ flakes. As with the HeLa cells, the macrophage cells internalized both nanographene sizes, again, with no obvious difference in the quantity of internalized material with respect to NGO flake size. Here again, the uptake happens in a many-particle fashion and inside the vesicles it is possible to see that the size of both samples match the measured values.

A very interesting observation was clearly visible in the morphology of the macrophages after they were incubated with the nanographene oxide for 48 hours. These cells are phagocytic white blood cells, widely distributed in the vertebrate body, and form part of the innate immune system. They are the first cells that respond to infectious agents such as viruses, toxins and
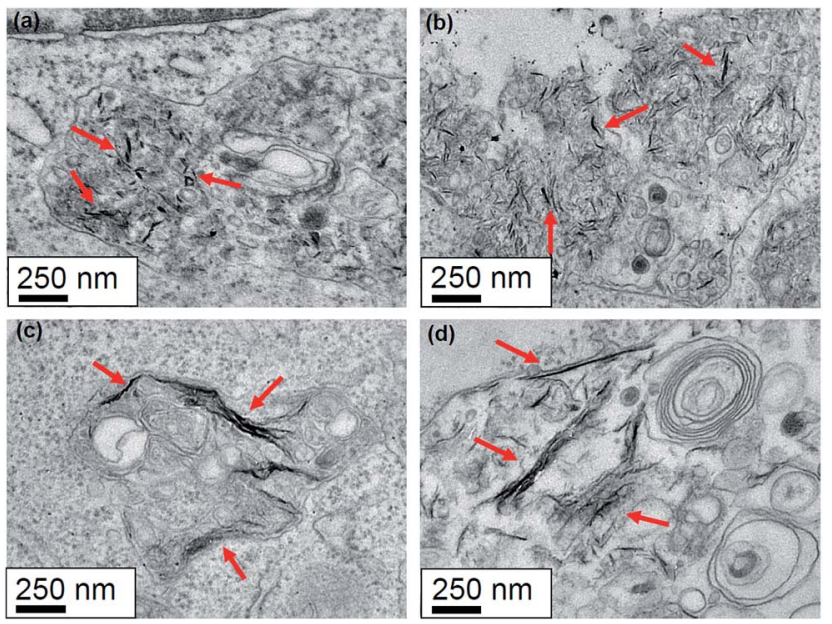

Fig. 6 Panels (a) and (b) show TEM images of a macrophage cells incubated with the $89 \mathrm{~nm} \mathrm{NGO}$ sample for 12 and $48 \mathrm{~h}$, respectively. In (c) and (d) a macrophage cell incubated with the $277 \mathrm{~nm}$ for 12 and 48 $h$, respectively. In the TEM images one can see that both sizes are internalized by HeLa cell as highlighted by the arrows.

most certainly also nanoparticles. These cells are able to ingest and kill harmful microorganisms by producing a variety of toxic chemicals and powerful degradative enzymes, which takes place inside vacuoles (phagosomes). ${ }^{38}$

In our observations, the macrophage cell line showed the formation of large vacuoles inside of which resided NGO flakes (Fig. 7). The cells were also found to increase in size. In comparison to the control samples and the 12 hours incubation, none of these changes could be observed. Fig. 7(a) shows the control sample without the presence of NGO. Fig. 7(b) and (c) show the macrophages incubated with the $89 \mathrm{~nm}$ nanographene oxide flakes for 12 and 48 hours respectively. The presence of big vacuoles after 48 hours incubation are easily observed and occupy enormous volumes of the cytoplasm. At a higher magnification the nanographene oxide can be identified inside the vacuoles. The same pattern is observed in Fig. 7(d) and (e) where the macrophages were incubated with the $277 \mathrm{~nm}$ NGO flakes for 12 and 48 hours incubation periods respectively.

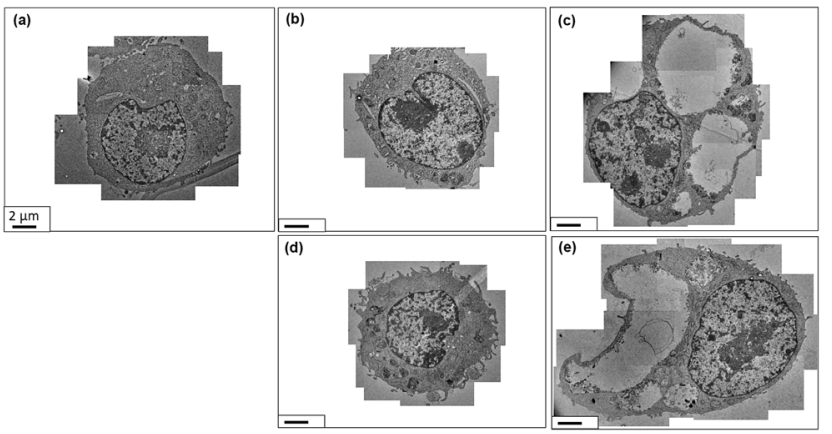

Fig. 7 Panel (a) shows the macrophages grown without NGO. Panels (b) and (c) show the macrophages incubated with the $89 \mathrm{~nm}$ graphene oxide for 12 and 48 hours, respectively. Cells are incubated with the $277 \mathrm{~nm} \mathrm{NGO}$ flakes for 12 and 48 hours and shown in panels (d) and (e), respectively. 
No differences in the uptake can be correlated with the size of the nanographene oxide.

The control samples were cultivated for the same time as those incubated with NGO flakes, however only cells who were in an environment with nanographene oxide flakes present showed the formation of vacuoles. These vacuoles only occur after longer exposure times to the nanographene oxide flakes. It is not clear why the macrophages changes the internal structures after 48 hours incubation, none-the-less our data does suggest it is the NGO flakes that trigger this response.

The formation of large vacuoles on macrophages has been observed before when these cells were infected with certain pathogens, however no detailed explanation with regards their initiation was provided. ${ }^{39}$ It is known that some molecules such as lipopolysaccharide (LPS), which is an integrant of outer membrane of Gram-negative bacteria, is a strong activator of macrophages and can induce the formation of large vacuolar structures. ${ }^{40}$ To our knowledge the cascade of events responsible to trigger the vacuolization has not been fully clarified yet, but previously published results suggest that its origin is related with other organelles such as plasma membrane, Golgi apparatus and endoplasmic reticulum ${ }^{40}$ as well as the p38 mitogenactivated protein kinase. ${ }^{41}$

The formation of vacuoles can be observed only after $48 \mathrm{~h}$ incubation and in the macrophage cell line only. The data suggests that both sizes of NGO trigger the formation of vacuoles and no size dependence can be observed in this case. Despite recently published studies investigated the interactions of macrophages with NGO were performed ${ }^{36,42}$ no formation of such large vacuoles was observed. In order to obtain further information if the NGO nanoparticles were inducing the formation of the vacuoles, a set of iron oxide nanoparticles at $10 \mu \mathrm{g} \mathrm{mL} \mathrm{m}^{-1}$ were incubated with the macrophages for 12,24 and 48 hours and the cells were imaged. The results shown in Fig. S8 in the ESI, $\dagger$ shows that the iron oxide nanoparticles are internalized and suggest that the nanoparticles are inside membrane-like structures like the NGO nanoparticles. However, the data with the iron oxide nanoparticles do not show cells with large vacuoles in any of the incubation times studied. This also suggests that not all types of nanoparticles trigger the formation of vacuoles. Although more studies must be conducted in order to better understand the processes involved in the observed cellular behavior, our data suggest that the NGO might be responsible for the biological signal which triggers the formation of increased vacuoles after 48 hours incubation.

\section{Conclusion}

The toxicity data assessed using NGO flakes with two distinct size distributions across two cell lines (HeLa cells and macrophages) suggest size can affect toxicity. For incubation times of 12 hours the small flakes $(89 \mathrm{~nm})$ show no difference with respect to concentration $\left(1,10\right.$ and $\left.100 \mu \mathrm{g} \mathrm{mL}{ }^{-1}\right)$, whereas for the larger flakes $(277 \mathrm{~nm})$ a reduction in viability is observed for the highest concentrations used ( 10 and $100 \mu \mathrm{g} \mathrm{mL}{ }^{-1}$ ). After 48 hours incubation a decrease in viability is observed when the NGO flake concentration increased for both cell lines. In the case of a longer incubation period, the sizes of the nanographene oxide seem to have little or no influence in the reduction of cell viability. The TEM investigations confirmed that the NGO flakes are internalized by both cell lines regardless of the incubation period. Moreover, no obvious difference in the uptake could be observed between the different sizes of the NGO. The uptake studies suggests that the macrophage cells undergo a morphology change after 48 hours incubation with NGO flakes regardless of size. This morphological change leads to the formation of large vacuoles containing NGO flakes. Future studies might better help understand this process. Our results provide valuable initial information on the cytotoxicity of NGO flakes with respect to flake size as well as cell linedependent morphological changes for extended incubation periods.

\section{Notes and references}

1 S. Dasgupta, T. Auth and G. Gompper, Nano Lett., 2014, 14, 687.

2 A. Albanese, P. S. Tang and W. C. W. Chan, Annu. Rev. Biomed. Eng., 2014, 14, 1.

3 R. Twarock and T. Keef, Microbiol. Today, 2010, 37, 24.

4 P. Cermelli, G. Indelicato and R. Twarock, Discrete and Topological Models in Molecular Biology Natural Computing Series, 2014, p. 217.

5 C. M. Feldherr, R. E. Lanford and D. Akin, Proc. Natl. Acad. Sci. U. S. A., 1992, 15, 11002.

6 Y. Xia, et al., Adv. Mater., 2007, 15, 353.

7 A. P. Alivisatos, Science, 1996, 271, 933.

8 R. Elghanian, J. J. Storhoff, R. C. Mucic, R. L. Letsinger and C. A. Mirkin, Science, 1997, 227, 1078.

9 L. R. Hirsch, et al., Proc. Natl. Acad. Sci. U. S. A., 2003, 100, 13549.

10 Y. Pan, S. Neuss, A. Leifert, M. Fischler, F. Wen, U. Simon, G. Schmid, W. Brandau and W. Jahnen-Dechent, Small, 2007, 3(11), 1941.

11 S. Tomic, et al., PLoS One, 2014, 9(5), e96584.

12 W. Jiang, B. Y. S. Kim, J. T. Rutka and W. C. W. Chan, Nat. Nanotechnol., 2008, 3, 145.

13 J. Gao, L. Wang, S. Kang, L. Zhao, M. Ji, C. Chen, Y. Zhao, R. Zhou and J. Li, Nanoscale, 2014, 6, 12828.

14 K. S. Novoselov, A. K. Geim, S. V. Morozov, D. Jiang, Y. Zhang, S. V. Dubonos, I. V. Grigorieva and A. A. Firsov, Science, 2004, 306, 666.

15 C. Lee, X. Wei, J. W. Kysar and J. Hone, Science, 2008, 321, 385.

16 S. Stankovich, D. A. Dikin, G. H. B. Dommett, K. M. Kohlhaas, E. J. Zimney, E. A. Stach, R. D. Pine, S. T. Nguyen and R. S. Ruoff, Nature, 2006, 442, 282.

17 K. S. Novoselov, A. K. Geim, S. V. Morozov, D. Jiang, M. I. Katsnelson, I. V. Grigorieva, S. V. Dubonos and A. A. Firsov, Nature, 2005, 438, 197.

18 C. Chung, Y.-K. Kim, D. Shin, S.-R. Ryoo, B. H. Hong and D.-H. Min, Acc. Chem. Res., 2013, 26, 2211.

19 Z. Liu, J. T. Robinson, X. Sun and H. Dai, J. Am. Chem. Soc., 2008, 130, 10876. 
20 X. Sun, Z. Liu, K. Welsher, J. T. Robinson, A. Goodwin, S. Zaric and H. Dai, Nano Res., 2008, 1, 203.

21 Y. Wang, Z. Li, D. Hu, C. T. Lin, J. Li and Y. Lin, J. Am. Chem. Soc., 2010, 132, 9274.

22 M. Vila, M. T. Portolés, P. A. A. P. Marques, M. J. Feito, M. C. Matesanz, C. Ramírez-Santillán, G. Gonçalves, S. M. A. Cruz, A. Nieto and M. Vallet-Regi, Nanotechnology, 2012, 23, 465103.

23 B. Chen, M. Liu, L. Zhang, J. Huang, J. Yao and Z. Zhang, J. Mater. Chem., 2011, 21, 7736.

24 Y. Wang, Z. Li, J. Wang, J. Li and Y. Lin, Trends Biotechnol., 2011, 29, 205.

25 Z. Liu, J. T. Robinson, X. Sun and H. Dai, J. Am. Chem. Soc., 2008, 130, 10876.

26 L. M. Zhang, Z. X. Lu, Q. H. Zhao, J. Huang, H. Shen and Z. J. Zhang, Small, 2011, 7, 460.

27 G. Gonçalves, M. Vila, M.-T. Portolés, M. Vallet-Regi, J. Gracio and P. A. A. P. Marques, Adv. Healthcare Mater., 2013, 2, 1072.

28 L. de Marzi, L. Ottaviano, F. Perrozzi, M. Nardone, S. Santucci, J. de Lapuente, M. Borras, E. Treossi, V. Palermo and A. Poma, J. Biol. Regul. Homeostatic Agents, 2014, 28(2), 281.

29 K. Wang, J. Ruan, H. Song, J. Zhang, Y. Wo, S. Guo and D. Cui, Nanoscale Res. Lett., 2011, 6, 1.

30 X. Zhang, J. Yin, C. Peng, W. Hu, Z. Zhu, W. Li, C. Fan and Q. Huang, Carbon, 2011, 29, 986.

31 Y. Liu, J. Wu, Y. Wang, X. Yang, R. Yang, B. Wang, J. Yang and N. Zhang, Sci. Rep., 2013, 3, 1.
32 D. Sahu, G. M. Kannan and R. Vijayaraghavan, Carbon black particle exhibits size dependent toxicity in human monocytes, Int. J. Inflammation, 2014, 2014, 1.

33 H. Kong, Y. Zhang, Y. Li, Z. Cui, K. Xia, Y. Sun, Q. Zhao and Y. Zhu, Int. J. Mol. Sci., 2013, 14, 22529.

34 W. S. Hummers and R. E. Offeman, J. Am. Chem. Soc., 1958, 80, 1339.

35 L. L. Staudenmaier, Ber. Dtsch. Chem. Ges., 1898, 31, 1481.

36 J. Linares, M. C. Matesanz, M. Vila, M. J. Feito, G. Gonçalves, M. Vallet-Regí, P. A. A. P. Marques and M. T. Portolés, ACS Appl. Mater. Interfaces, 2014, 6, 13697.

37 Q. Mu, G. Su, L. Li, B. O. Gilbertson, L. H. Yu, Q. Zhang, Y. P. Sun and B. Yan, ACS Appl. Mater. Interfaces, 2012, 4, 2259.

38 K. M. Murphy, P. Travers and M. Walport, in Janeway's Immunobiology, Garland Science, Taylor \& Francis Group, LLC, New York, 8th edn, 2011, pp. 3-6.

39 M. S. Gomes, S. Paul, A. L. Moreira, R. Appelberg, M. Rabinovitch and G. Kaplan, Infect. Immun., 1999, 67, 3199.

40 K. Yoshida, M. Ono and H. Sawada, J. Endotoxin Res., 1999, 5, 127.

41 F. Hassan, S. Islam, N. Koide, M. M. Mu, H. Ito, I. Mori, T. Yoshida and T. Yokochi, Microbiol. Immunol., 2004, 48, 807.

42 F. Feito, M. Vila, M. C. Matesanz, J. Linares, G. Gonçalves, P. A. A. P. Marques, M. Vallet-Regí, J. M. Rojo and M. T. Portolés, J. Colloid Interface Sci., 2014, 432, 221. 